\section{PSYCAUSE}

Revue scientifique étudiante de

l'École de psychologie de l'Université Laval

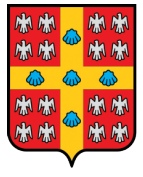

UNIVERSITÉ LAVAL

Faculté des sciences sociales École de psychologie

SEPTEMBRE 2018 - VOL. $8 \mathrm{~N}^{\circ} 2$

\title{
LIENS ENTRE LA SOLITUDE ET LES TYPES D'UTILISATION DE FACEBOOK
}

Mélissa BAILLARGEON ${ }^{1, *}$, Anne-Hélène HARRISSON ${ }^{1}$, Joanie MERCIER ${ }^{1}$ \& Marie-Claude RICHARD ${ }^{1}$

1 École de psychologie, Université Laval, Québec, Canada

*melissa.baillargeon.1@ulaval.ca

\section{Pour citer l'article}

Baillargeon, M., Harrisson, A.-H., Mercier, J., \& Richard, M.-C. (2018). Liens entre la solitude et les types d'utilisation de Facebook. Psycause: Revue scientifique étudiante de l'École de psychologie de I'Université Laval, 8(2), 23-25. 
Sarkadi, A., Kristiansson, R., Oberklaid, F., \& Bremberg, S. (2008). Fathers' involvement and children's developmental outcomes: A systematic review of longitudinal studies. Acta paediatrica, 97, 153-158. doi:10.1111/ j.1651-2227.2007.00572.x

Pleck, J. H. (2012) Integrating father involvement in parenting research. Parenting, 12, 243253. doi:10.1080/15295192.2012.683365
Turcotte, G., Dubeau, D., Bolté, C., \& Paquette, D. (2001). Pourquoi certains pères sont-ils plus engagés que d'autres auprès de leurs enfants? Une revue des déterminants de l'engagement paternel. Revue canadienne de psychoéducation, 30, 65-93. Récupéré sur https://unites.uqam.ca/grave/prospere/ pages/pdf/Turcotte_Dubeau.pdf

\section{LIENS ENTRE LA SOLITUDE ET LES TYPES D'UTILISATION DE FACEBOOK}

Mélissa BAILLARGEON, Anne-Hélène HARRISSON, Joanie MERCIER \& Marie-Claude RICHARD École de psychologie, Université Laval, Québec, Canada

\section{Introduction}

Plusieurs travaux ont tenté de clarifier les liens entre la solitude et l'utilisation des réseaux sociaux en ligne sans parvenir à un consensus. Par exemple, les études de la méta-analyse de Song et coll. (2014)—qui s'intéressent à la quantité de temps passé sur Facebook, à l'usage excessif ou compulsif de Facebook, et celles comparant les utilisateurs aux nonutilisateur de Facebook-tendent à montrer un lien avec une augmentation de la solitude. Par contre, d'autres études de cette même méta-analyse tendent à montrer un lien entre I'intensité de l'utilisation de Facebook (c.-à-d. le nombre d'amis, le temps quotidien passé sur Facebook, la quantité de contenu créé, le nombre de communications directes entretenues sur Facebook et leur attitude par rapport à Facebook) et une diminution de la solitude. Une explication de ces résultats divergents est que les études ne prennent pas en compte les types d'utilisation de Facebook (c.-à-d. actif social, actif non social et passif; voir le Tableau 1 tiré de Gerson, Plagnol \& Corr, 2017). De plus, certains auteurs ont exploré la relation entre les types d'utilisation de Facebook et la perception de soutien social (Seo, Kim \& Yang, 2016). II ressort donc de ce qui précède que les personnes utilisant davantage Facebook de façon active ont une plus grande perception de soutien social que ceux utilisant les autres types d'utilisation. Les objectifs de cette étude consistent à déterminer si : (a) les types d'utilisation de Facebook sont associés au sentiment de solitude; et si (b) la perception de soutien social contribue à expliquer cette relation.

\section{Méthode}

Trois cent onze adultes québécois utilisateurs de Facebook âgés de 18 à 29 ans $(M=22,59 ; \dot{E}-T=$ 2,68 ) ont rempli le questionnaire de 41 questions à partir de l'application web LimeSurvey.

L'Échelle de solitude de l'Université Laval (ÉSUL; de Grâce, Joshi \& Pelletier, 1993), la version francophone du UCLA Loneliness Scale, a été utilisée pour évaluer la solitude. Cette échelle comprend 20 énoncés autodescriptifs pour lesquels le participant indique la fréquence d'apparition d'un sentiment spécifique de solitude dans sa vie (Russell, 1996). La souséchelle Soutien familial du Multidimensional Scale of Perceived Social Support (MSPSS), adaptée par Frison et Eggermont (2016), a été utilisée pour évaluer la perception de soutien social sur Facebook (Zimet, Powell, Farley, Werkman \& Berkoff, 1990). Le Passive and Active Use Measure (PAUM; Gerson et coll., 2017) a été utilisé pour évaluer les types 
d'utilisation de Facebook. Le PAUM comprend 13 items évaluant I'utilisation de Facebook selon trois sous-échelles: active sociale, active non sociale et passive. Le score de chaque sous-échelle s'obtient en additionnant les scores des items qu'elle contient.

\section{Résultats et discussion}

Les résultats de la régression linéaire multiple montrent que l'utilisation passive de Facebook contribue significativement à prédire la solitude. Cela suggère que plus une personne utilise Facebook de façon passive, plus elle se sent seule. Ces données appuient celles de certains auteurs ayant observé ce lien entre une utilisation passive des réseaux sociaux et un sentiment plus élevé de solitude (Gardner, Pickett \& Knowles, 2005). Les résultats de la régression indiquent que l'utilisation active (sociale et non sociale) de Facebook n'est pas liée au sentiment de solitude. Cette absence de lien pourrait être expliquée par une mauvaise opérationnalisation du PAUM dans le classement des items. Effectivement, le PAUM ne distingue pas le caractère personnel (c.-à-d. des photos prises par eux-mêmes) ou impersonnel (c.-à-d. des vidéos trouvées en ligne) du contenu partagé.
Également, la motivation derrière I'utilisation de Facebook (compenser vs compléter les relations sociales) pourrait influencer le sentiment de solitude (Nowland, Necka \& Cacioppo, 2017).

Les analyses révèlent qu'il n'y a pas de relation significative entre la perception de soutien social et la solitude, ni entre l'utilisation passive de Facebook et la perception de soutien social. Bien que les résultats de la présente étude indiquent que la perception de soutien social n'est pas un modérateur, d'autres études ont obtenu des résultats appuyant un modèle de modération. En effet, Seo et coll. (2016) ont trouvé un rôle modérateur de la perception de soutien social entre des mesures d'utilisation de Facebook (nombre d'interactions et temps moyen pour recevoir un commentaire) et la solitude. Également, il est possible que d'autres variables que la perception de soutien social influencent la relation entre I'utilisation de Facebook et la solitude. Nowland et coll. (2017) remarquent que les femmes utilisent davantage les réseaux sociaux de façon active sociale que les hommes. Ainsi, il serait possible que le sexe soit une variable influençant les types d'utilisation de Facebook et, ultimement, le niveau de solitude.

\section{Tableau 1}

Définition et types d'activité selon le type d'usage de Facebook (Gerson, Plagnol \& Corr, 2017)

\begin{tabular}{|c|c|c|}
\hline Type d'usage & Définition & Types d'activité \\
\hline Actif social & $\begin{array}{l}\text { Création de contenu } \\
\text { avec communication } \\
\text { directe avec les autres } \\
\text { utilisateurs. }\end{array}$ & $\begin{array}{l}\text { Publier des statuts; } \\
\text { Commenter diverses publications; } \\
\text { Interagir par l'intermédiaire de la messagerie } \\
\text { instantanée; } \\
\text { Publier des photos; } \\
\text { Naviguer activement sur le fil d'actualité (aimer } \\
\text { ou commenter des publications). }\end{array}$ \\
\hline Actif non social & $\begin{array}{l}\text { Création de contenu } \\
\text { sans communication } \\
\text { directe avec les autres } \\
\text { utilisateurs. }\end{array}$ & $\begin{array}{l}\text { Identifier des photos; } \\
\text { Publier et identifier des vidéos; } \\
\text { Créer un événement ou indiquer la participation à } \\
\text { un évènement. }\end{array}$ \\
\hline Passif & $\begin{array}{l}\text { Consommation de } \\
\text { contenu sans création } \\
\text { de contenu et sans } \\
\text { communication } \\
\text { directe avec les autres } \\
\text { utilisateurs. }\end{array}$ & $\begin{array}{l}\text { Regarder des photos; } \\
\text { Naviguer passivement sur le fil d'actualité (sans } \\
\text { aimer ou commenter de publications); } \\
\text { Regarder le profil des contacts; } \\
\text { Vérifier, par le biais de Facebook, ce que les autres } \\
\text { utilisateurs font. }\end{array}$ \\
\hline
\end{tabular}




\section{Références}

de Grâce, G. R., Joshi, P., \& Pelletier, R. (1993). L'Échelle de solitude de l'Université Laval (ESUL): Validation canadienne-française du UCLA Loneliness Scale. Revue canadienne des sciences du comportement, 25, 12-27. doi:10.1037/h0078812

Frison, E., \& Eggermont, S. (2016). Exploring the relationships between different types of Facebook use, perceived online social support, and adolescents' depressed mood. Social Science Computer Review, 34, 153-171. doi:10.1177/0894439314567449

Gardner, W. L., Pickett, C. L., \& Knowles, M. (2005). Social snacking and shielding: Using social symbols, selves, and surrogates in the service of belonging needs. Dans K. D. Williams, J. P. Forgas, \& W. von Hippel (Eds.), The social outcast: Ostracism, social exclusion, rejection, and bullying (pp. 227-242). New York, NY: Psychology Press.

Gerson, J., Plagnol, A. C., \& Corr, P. J. (2017). Passive and active Facebook use measure (PAUM): Validation and relationship to the reinforcement sensitivity theory. Personality and Individual Differences, 117, 81-90. doi:10.1016/j.paid.2017.05.034
Nowland, R., Necka, E. A., \& Cacioppo, J. T. (2017). Loneliness and social internet use: Pathways to reconnection in a digital world? Perspectives on Psychological Science, 13, 70-87. doi:10.1177/1745691617713052

Russell, D. (1996). UCLA Loneliness Scale (Version 3): Reliability, validity and factor structure. Journal of Personality Assessment, 66, 20-40. doi:10.1207/s15327752jpa6601_2

Seo, M., Kim, J., \& Yang, H. (2016). Frequentinteraction and fast feedback predict perceived social support: Using crawled and self-reported data of Facebook users. Journal of ComputerMediated Communication, 21, 282-297. doi:10.1111/jcc4.12160

Song, H., Zmyslinski-Seelig, A., Kim, J., Drent, A., Victor, A., Omori, K., \& Allen, M. (2014). Does Facebook make you lonely?: A meta analysis. Computers in Human Behavior, 36, 446-452. doi:10.1016/j.chb.2014.04.011

Zimet, G.D.,Powell,S.S., Farley, G. K., Werkman,S.,\& Berkoff, K. A. (1990). Psychometric characteristics of the multidimensional scale of perceived social support. Journal of Personality Assessment, 55, 610-617. doi:10.1080/00223891.1990.9 67409 